PO-0091 5 YEAR OLD GIRL WITH DISPROPORTIONATE SHORT STATURE WITH FAILURE TO THRIVE AND DYSMORPHISM: RARE CASE OF SCHEIE SYNDROME

1J Cheriathu, ${ }^{2} \mathrm{~L}$ Jenny John, 'M Shamseldeen. 'Pediatrics, Gulf Medical University, Ajman, United Arab Emirates; ${ }^{2}$ Pharmacology, Gulf Medical University, Ajman, United Arab Emirates

\subsection{6/archdischild-2014-307384.760}

Background Hurler-Scheie Syndrome is an autosomal recessive mucopolysaccharidois resulting in reduced activity of $\alpha$-L-iduronidase with accumulation of heparin and dermatan sulphate. Scheie syndrome represents less severely affected form with varied clinical features.

Case Report 5 year female, first born child of 2nd degree consanguineous Egyptian parents, referred for short stature presented with following features.

On examination:

Length: $88 \mathrm{~cm}$; Upper: Lower segment=1:1.3; weight: 12.3 $\mathrm{kg}$; Head circumference: $51 \mathrm{~cm}$

Protuberant abdomen, mild proptosis, enlarged skull, prominent forehead, low set ears, simian crease, clawed hand, mild kyphosis, strabismus, normal IQ, hepatomegaly.

Investigation: USG: hepatomegaly, Karyotyping normal Mild delay bone age; Elevated TSH, low T4; tissue transglutaminase normal; sweat chloride test normal; ammonia, lactate normal; growth hormone assay normal; Deficient enzyme in fibroblast confirmed diagnosis of Scheie syndrome

Discussion Scheie syndrome is an autosomal recessive, rare lysosomal storage disease, with skeletal deformities and motor delay; described first in 1972; caused by mutations in IDUA gene (4p16.3) leading to partial deficiency in alpha-L-iduronidase enzyme and lysosomal accumulation of dermatan and heparan sulfate. Genetic testing is available. Antenatal diagnosis done by measurement of enzymatic activity in chorionic villus/ amniocytes and by genetic testing (if disease-causing mutation is known). Genetic counselling is recommended. Management is multidisciplinary including physiotherapy (to maintain range of movement); bone marrow or umbilical cord blood transplant (to preserve neurocognition, improve somatic disease and increase survival). Enzyme replacement therapy slows disease progression.

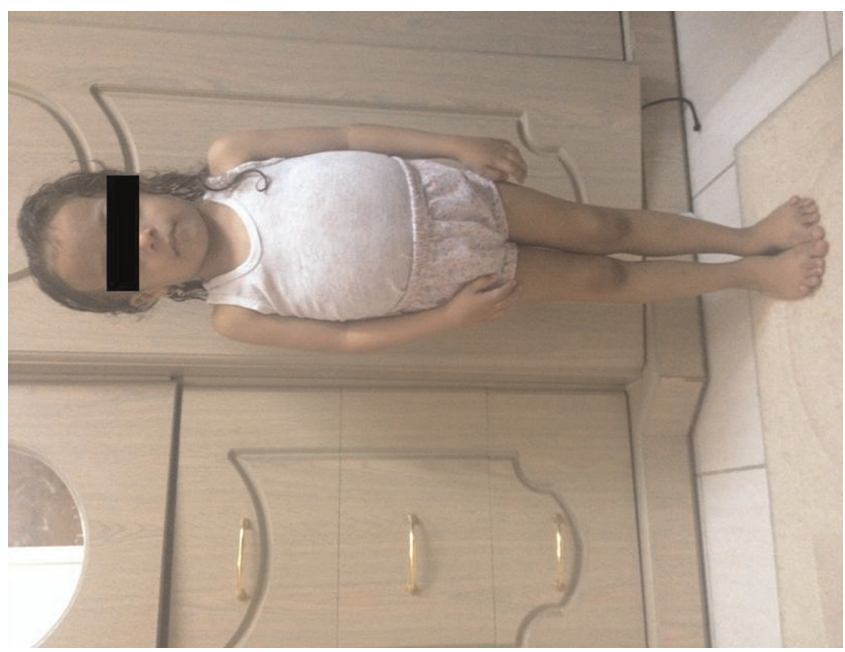

Abstract P0-0091 Figure 1
Conclusion Scheie syndrome should be considered in differential diagnosis of disproportionate short stature with dysmorphism, failure to thrive with normal IQ.

\section{PO-0092 GC-MS IN DIAGNOSIS OF BEHAVIOURAL ABNORMALITIES AND DYSMORPHISM : A CASE SERIES}

I Panigrahi, P Goyadi, N Goya, RK Marwaha. Pediatrics, Postgraduate Institute of Medical Education and Research (PGIMER), Chandigarh, India

\subsection{6/archdischild-2014-307384.761}

Background Several metabolic disorders can present with behavioural abnormalities or autism. There may or maynot be associated seizures or acute metabolic decompensation. Gas chromatography and mass spectrometry (GC-MS) is a modality for non-invasive testing for some of these disorders, especially organic acidurias. Children with dysmorphism, malformations and metabolic disorders are followed up in Genetic Clinic.

Aims We present here 7 cases of metabolic disorders diagnosed on GC-MS testing on urine and on follow up in the Genetic Clinic.

Methods The cases of suspected metabolic disorders were worked up through the outpatient clinic or during admission in Genetic ward. Patients seen in 11 year (Jan. to Dec. 2014) were included in the study.

Results There were 4 cases of methylmalonic acidemia (MMA), one case each of 3-methylglutaconic aciduria, didhydrolipoyldehydrogenase (DLD) deficiency and MMA with homocystinuria. The cases with MMA presented with developmental delay, hyperactivity and behavioural abnormalies. There was subtle dysmorphism on clinical evaluation. The child with MMA with homocystinuria presented with mental retardation and prominent forehead. $\mathrm{He}$ was admitted earlier with an episode of DVT and detailed evaluation led to the diagnosis. The child with 3-methyl-glutaconic aciduria presented with behavioural abnormalities.

Conclusions MMA was found to be a commoner metabolic disorder and treatable. A high index of suspicion is needed in diagnosis and an early diagnosis can improve the outcomes in patients with metabolic disorder.

\section{PO-0093 FAMILIAL PURE GONADAL DYSGENESIS}

S Dalili, S Kohmanaee, A Hassanzadeh Rad. Pediatrics Growth Disorders Research Center 17th Shahrivar Hospital School of Medicine Guilan University of Medical Sciences Rasht Iran, Guilan University of Medical Sciences, Rasht, Iran

\subsection{6/archdischild-2014-307384.762}

Introduction Gonadal dysgenesis in females which would be presented with $46 \mathrm{XX}$ is characterised by the presence of primary amenorrhea with or without normal secondary sexual characteristics. It is commonly described without normal secondary sexual characteristics such as breast development. In this study, we aimed to report a familial pure gonadal dysgenesis with and without normal secondary sexual characteristics.

Case presentations In a family consisting 9 siblings of non-consanguineous parents, three sisters (age 22, 17 and 13 years) presented with complaints of amenorrhoea. The 13 years old girl mentioned no secondary characteristics of puberty. The 17 years old girl showed secondary characteristics of puberty without previous history of hormone therapy. Also, the 22 years old girl showed secondary characteristics and clinicians recommended hormone therapy after marriage for her infertility and even delivered a baby. The elder two sisters had a normal female 